\title{
X-ray Texture Analysis of Thin Films by the Reflection Method: Intermediate Regime in Defocusing Corrections
}

\author{
By D. Chateigner, P. Germi and M. Pernet \\ Laboratoire de Cristallographie, Centre National de la Recherche Scientifique, associé à l'Université \\ Joseph Fourier, BP166, 38042 Grenoble CEDEX 09, France
}

(Received 6 April 1993; accepted 13 August 1993)

\begin{abstract}
This paper gives the angular domains of validity for corrections of experimental data obtained from the texture analysis of thin films, multilayers and covered substrates by the Schulz reflection technique. The behaviours of defocusing curves versus material constants are given as examples and their effects on correction curves are shown. The correction formulas for characteristic types of multilayers are also deduced and are illustrated for one example.
\end{abstract}

\section{Introduction}

The most commonly used technique for texture analysis is the Schulz reflection method (Schulz, 1949), which is now also widely employed for thin-film analysis. In this technique, a sample without preferred orientation of crystallites is needed for the correction of experimental intensities. However, the elaboration of untextured thin-layer compounds is practically unrealizable owing to effects such as growth and form anisotropy, layer interactions etc. In those cases, special corrections for defocusing are needed and these have recently been detailed (Chateigner, Germi $\&$ Pernet, 1992). When the incidence of the beam versus the Bragg and tilt angles, 0 and $\varphi$, was considered, two relations were deduced that give limits for intensity corrections, assuming the sample to be a film if $\varphi<\operatorname{arcos}\left(e / \xi_{m}\right)$ and $\theta>\arcsin \left(e / \xi_{m}\right)$, where $e$ is the thickness of the film and $\xi_{m}$ is the penetration depth of the radiation, which is defined by the ratio $1 / 2 \mu$, where $\mu$ is the linear absorption coefficient of the corresponding material. These limitations, taken separately, restrict slightly the applicability of the proposed correction but are more restrictive when combined, as discussed below.

With multilayer texture analysis, developments could also be ruled out in special cases where the sample configuration plays an important role. Some examples are described here in order to demonstrate the employment of the general formulation.

\section{Theory and examples}

Thin films

For film texture analysis by the Schulz reflection method, the defocusing of the diffracted intensity, $\operatorname{Id}^{f}(\varphi, \theta)$, is related to the experimental bulk one, $\operatorname{Id}^{b}(\varphi, \theta)$, by

$$
\operatorname{Id}^{f}(\varphi, \theta)=e \operatorname{Id}^{b}(\varphi, \theta) / \xi_{m} \sin \theta \cos \varphi .
$$

This formulation is a good approximation of the previous one deduced from equations (4) and (5) of Schulz (1949) in the case of thin films (in the sense of the definition of $\xi_{m}$ ). It was first employed for intensity corrections in the texture analysis of polycrystalline silicon films by Wenk, Sintubin, Huang, Johnson \& Howe (1990).

Fig. $1(a)$ is a view of the scattering plane at $\varphi=0$, where the path of the beam, $x$, into the sample is indicated. Equation (1) is only applicable when $x \leq \xi_{m}$ with

$$
x=p / \sin \theta .
$$

As shown in Fig. $1(b)$, when $\varphi \neq 0$,

$$
p=e / \cos \varphi
$$

in such a manner that the condition $x \leq \xi_{m}$ becomes

$$
e / \sin \theta \cos \varphi \leq \xi_{m},
$$

which defines the allowed domain in $\varphi$ or $\theta$ for (1). Since $|\sin \theta| \leq 1$ and $|\cos \varphi| \leq 1$, (4) always reduces the applicable domain of (1) more than the previously defined conditions (Chateigner et al., 1992). No specific film defocusing correction has to be made for a studied $h k l$ reflection when (even if $\varphi=0$ ) (4) is not fulfilled, leading to the expression of a limit layer thickness $e_{1}$ :

$$
e_{l}=\xi_{m} \sin \theta
$$

Hence, this later limit is a criterion for the choice of the correction applicable to a specific pole figure.

When the two terms of (4) are equal, both film and bulk irradiated under this incidence scatter the same 
intensity and (1) therefore gives $\operatorname{Id}^{f}(\varphi, \theta)=\operatorname{Id}^{b}(\varphi, \theta)$, the largest value of $\operatorname{Id}^{f}(\varphi, \theta)$. At that point, the tilt angle reaches the value $\varphi_{l}$ [deduced from (4)], above which the bulk defocusing has to be used.

Fig. 2 shows calculated defocusing curves for 103 reflections of $\mathrm{YBa}_{2} \mathrm{Cu}_{3} \mathrm{O}_{7}$ films at $0=16.44^{\circ}$ for different thicknesses. The experimental bulk curve was obtained on a randomly oriented powder of $\mathrm{YBa}_{2} \mathrm{Cu}_{3} \mathrm{O}_{7}$ for the same $\theta$, prepared by a classical solid-state reaction from powder oxides. The randomness of the powder was confirmed by a $\theta-20$ scan, where the ratios between peak intensities were in good agreement with theoretical powder pattern. The linear absorption coefficient has been estimated for $\mathrm{YBa}_{2} \mathrm{Cu}_{3} \mathrm{O}_{7}$ with parameters $a=3.825, b=3.886$ and $c=11.66 \AA$ (Reller, Bednorz \& Muller, 1989) with $\mathrm{Cu} K \alpha$ radiation. We found $\mu=1091.5 \mathrm{~cm}^{-1}$. It is clear from these curves that the defocusing is more pronounced for thicker films. The calculation (1) was

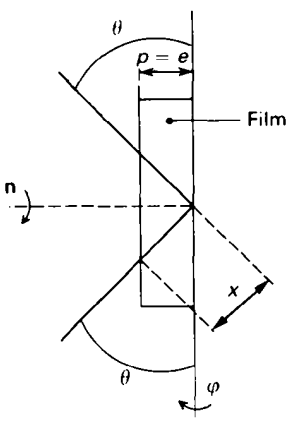

(a)

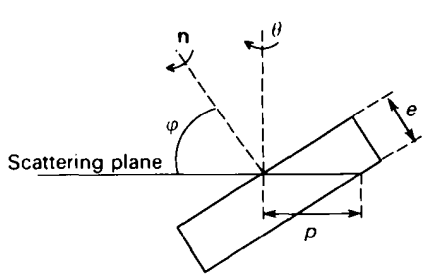

(b)
Fig. 1. Cross sections in the Schulz-reflection-method geometry of (a) the top view of the scattering plane at $\varphi=0 ;(b)$ the plane defined by the normal to the sample plane and the $\theta$ rotation axis, with $\varphi>0$.

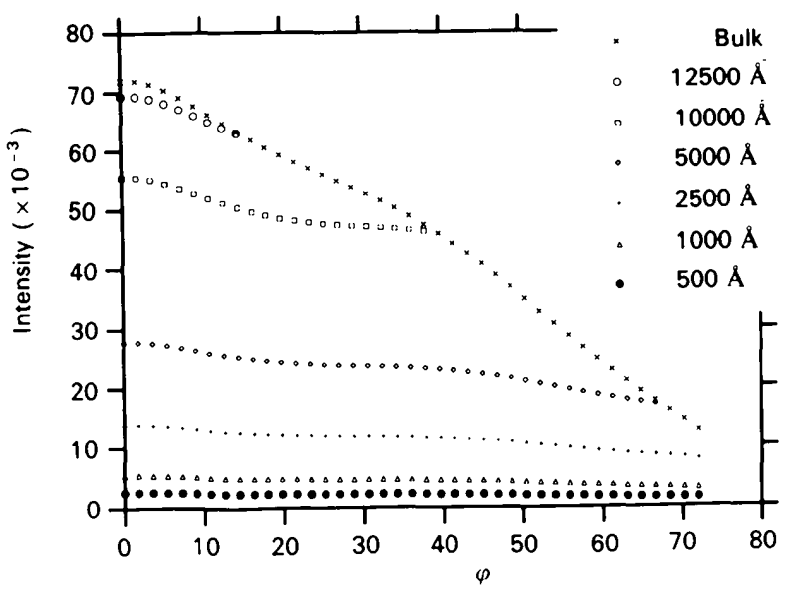

Fig. 2. Experimental bulk defocusing curve obtained on a randomly oriented sample and calculated film ones for different thicknesses of the layer for a $\mathrm{YBa}_{2} \mathrm{Cu}_{3} \mathrm{O}_{7} /$ substrate sample. $\theta$ was kept constant at $16.44^{\circ}$.
Table 1. Values of the limit layer thicknesses $\left(e_{t}\right)$ in $\mu \mathrm{m}$ for the principal Bragg peaks of a $\mathrm{YBa}_{2} \mathrm{Cu}_{3} \mathrm{O}_{7}$ film with $\mathrm{Cu} K \alpha$ radiation

$\begin{array}{lcccccccc}h k l & 001 & 002 & 003 & 0.12 & 103 & 005 & 113 & 006 \\ e_{l} & 0.305 & 0.605 & 0.91 & 1.09 & 1.295 & 1.515 & 1.58 & 1.815\end{array}$

done with the incorporation of (4) since for $\varphi$ 's greater than $\varphi_{l}$ the film defocusing curves become indistinguishable from ones for bulk material of infinite thickness.

For this material, (5) gives the different values of $e_{l}$ (Table 1). It is clear that different studied pole figures (different $\theta$ ) would necessitate different types of correction (for bulk, film or intermediate), depending on the layer thicknesses. As an example, a $1 \mu \mathrm{m}$ layer is treated as a bulk and as a film for the 003 and 113 pole figures, respectively. For a constant $\theta$ value and up to $\varphi_{l}$, there is no difference in the behaviour of the inverse correction curves obtained with different $e$ or $\xi_{m}$ parameters. This corresponds to the correction function $C(\varphi, \theta)=\operatorname{Id}^{f}(\varphi, \theta) / \operatorname{Id}^{f}(0, \theta)$ (Gale \& Griffiths, 1960 ; Tenckhoff, 1970), since then only $\cos \varphi$ remains in the defined ratio.

\section{Substrate}

For substrates, the correction has to be made through the expression for one covering layer (Chateigner et al., 1992):

$$
\operatorname{Id}^{s}(\varphi, \theta)=\operatorname{Id}^{\text {bs }}(\varphi, \theta) \exp [-2 \mu e /(\cos \varphi \sin \theta)],
$$

where $\operatorname{Id}^{s}(\varphi, \theta)$ and $\operatorname{Id}^{\mathrm{bs}}(\varphi, \theta)$ are the diffracted intensities for the covered and uncovered substrates, respectively, and $\mu$ is the linear absorption coefficient of the film material.

Owing to the $e / \cos \varphi$ ratio in the exponential term, the relation $\operatorname{Id}^{s}(\varphi, \theta)<\operatorname{Id}^{\mathrm{bs}}(\varphi, \theta)$ is always satisfied and there is no intermediate regime for intensity corrections. Fig. 3 shows the defocusing curves for an $\mathrm{MgO}$ substrate covered by a layer of $\mathrm{YBa}_{2} \mathrm{Cu}_{3} \mathrm{O}_{7}$ of varying thickness. The chosen reflection from the substrate is 200 at $\theta=21.47^{\circ}$. The bulk defocusing curve was obtained on a $97 \%$ pure and randomly oriented powder while the calculation (6) provides the curves for a covered substrate. Of course, for all $\varphi$ positions the diffracted intensity decreases with the thickness of the film, but the correction that has to be made to pole-figure values increases, resulting in a less extended allowable experimental domain. The pole figure is therefore less complete than for an uncovered substrate.

As an illustration, Table 2 shows $\varphi$ values $\varphi_{50}$ and $\varphi_{20}$, where the correction ratio $C(\varphi, \theta)$ equals 50 and $20 \%$, respectively, as functions of the thickness of the film. This numerical determination of $\varphi_{50}$ and $\varphi_{20}$ has been conducted for the previous example and shows how the pole figure is incomplete. In this example we 
Table 2. $\varphi_{50}$ and $\varphi_{20}$ values of the correction ratio $C(\varphi, \theta)$ for an $\mathrm{MgO}$ substrate covered by a $\mathrm{YBa}_{2} \mathrm{Cu}_{3} \mathrm{O}_{7}$ film of varying thickness e for the 200 reflection of $\mathrm{MgO}$ at $\theta=21.47^{\circ}$

$\begin{array}{lrccrrr}e(\mu \mathrm{m}) & 0 & 0.1 & 0.5 & 1 & 2 & 4 \\ \varphi_{50} & 63 & 62 & 58 & 52 & 44 & 36 \\ \varphi_{20} & >72 & >72 & 70 & 65 & 58 & 47\end{array}$

see that the experimental bulk-substrate reflection is no greater than $20 \%$ of its $\varphi=0$ value at only $\varphi \simeq 47^{\circ}$ for $e=4 \mu \mathrm{m}$ and of course is less at higher $\varphi$ 's. This small value compared with the uncovered substrate may have important consequences for the analysis of textures by the reflection method. This tendency is lowered as $e$ decreases but still remains. Fig. 4 shows the correction curves of such samples. Unlike with the correction of uncovered bulks, the variation of $\mu$ also produces different correction curves (Tenckhoff, 1970). The constant part at low $\varphi$ of such curves (Couterne $\&$ Cizeron, 1971) no longer exists, since the absorption by the film begins at $\varphi>0$. This effect is not clearly seen here because we use a thin slit aperture for detection with the $\mathrm{YBa}_{2} \mathrm{Cu}_{3} \mathrm{O}_{7}$ system, which suppresses the $C(\varphi, 0)=1$ part.

\section{Buffer layer}

For an intermediate layer, the combination of (6) and (1) gives

$$
\begin{aligned}
\operatorname{Id}^{\mathrm{bl}}(\varphi, \theta)= & {\left[e^{\prime} \operatorname{Id}^{\mathrm{bb}}(\varphi, \theta) / \xi_{m}^{\prime} \sin \theta \cos \varphi\right] } \\
& \times \exp (-2 \mu e / \sin \theta \cos \varphi)
\end{aligned}
$$

$\operatorname{Id}^{\mathrm{bl}}(\varphi, \theta)$ and $\operatorname{Id}^{\mathrm{bb}}(\varphi, \theta)$ are the corresponding diffracted intensities for the layer and the bulk, respectively, and primes denote the buffer-layer thickness and penetration depth.

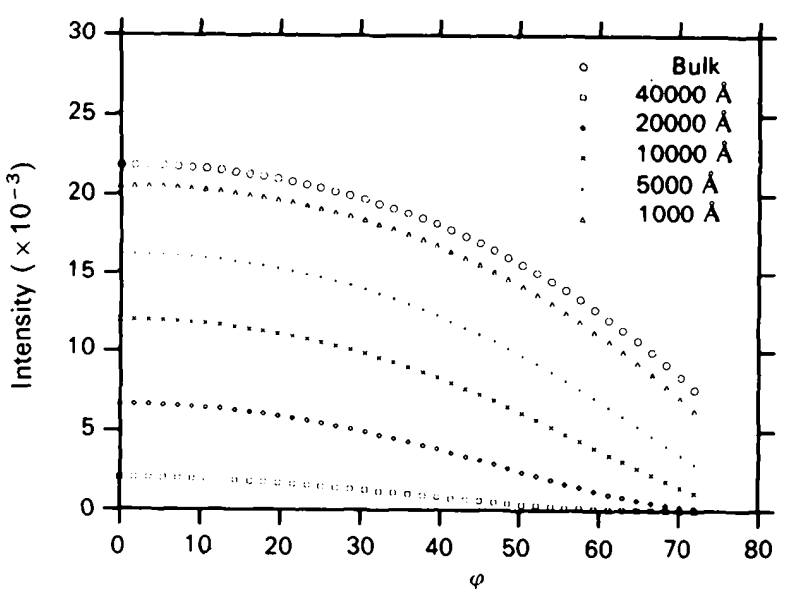

Fig. 3. Experimental bulk defocusing curves for the 200 reflection of $\mathrm{MgO}$ at $\theta=21.47^{\circ}$ and calculated ones for the same substrate covered by $\mathrm{YBa}_{2} \mathrm{Cu}_{3} \mathrm{O}_{7}$ films of various thicknesses.
The variation of the material parameters gives different solutions of $\operatorname{Id}^{\mathrm{bl}}(\varphi, \theta)<\mathrm{Id}^{\mathrm{bb}}(\varphi, \theta)$, but there is no analytical solution of this inequality. Nevertheless, the problem is by-passed directly during computation of the new defocusing curve. This point is illustrated in Fig. 5, where the thicknesses of a $\mathrm{YBa}_{2} \mathrm{Cu}_{3} \mathrm{O}_{7}$ film (Fig. 5a) and the YSZ (yttriastabilized zirconia) buffer layer (Fig. $5 b$ ) have been varied. The experimental bulk points were obtained on a random powder of nominal composition $\mathrm{Y}_{0.15} \mathrm{Zr}_{0.85} \mathrm{O}_{2}$ and are the same in the two figures since they are referred to the same reflection peak, 111 YSZ, at $\theta=15.02^{\circ}$. In Fig. $5(a)$, we took $e^{\prime}=10000 \AA$ and $\xi_{m}^{\prime}=7.72 \mu \mathrm{m}$, while, in Fig. $5(b), e=500 \AA$ and $\mu=0.10915 \mu \mathrm{m}^{-1}$. We see clearly from these figures and from (7) that the material parameters $e, \mu$ and $\xi_{m}^{\prime}$ on one hand and $e^{\prime}$ on the other hand have opposite effects on intensity.

It is necessary to discuss the influence of these parameters on the correction curves. For one type of reflection plane, whatever the values of $e^{\prime}$ and $\xi_{m}^{\prime}$, the correction remains identical as in film corrections (with the same limits in $\varphi_{l}$ ). This is not the case when $e$ and $\mu$ vary, such as in substrate corrections. Consequently, it is necessary to modify such curves for every combination of $e$ and $\mu$.

\section{Multilayers}

In the case of multilayers, a pole figure obtained from a separated Bragg peak referred to a single $j$ th layer has to be corrected by

$$
\begin{aligned}
\operatorname{id}_{j}^{\mathrm{bl}}(\varphi, \theta)= & {\left[e_{j} \mathrm{Id}_{j}^{\mathrm{bb}}(\varphi, \theta) / \xi_{m} \sin \theta \cos \varphi\right] } \\
& \times \exp \left(-2 \sum_{i=1}^{j-1} \mu_{i} e_{i} / \sin \theta \cos \varphi\right) .
\end{aligned}
$$

It is rare to have all layers of different compositions with independent Bragg peaks. Generally, multilayer

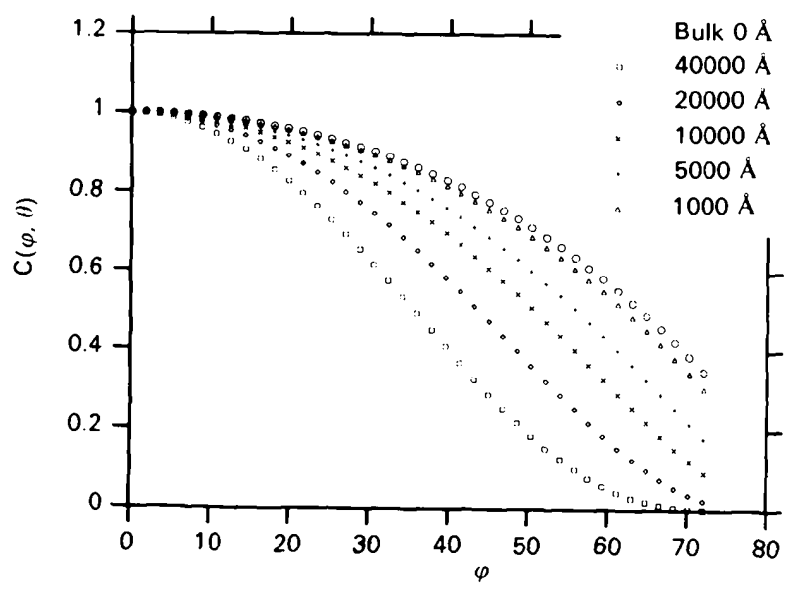

Fig. 4. Calculated correction curves, $C(\varphi, \vartheta)$, for the sample of Fig. 3. 
samples are composed of some different phases successively and periodically stacked. Of course, the contributions of the layers of the same phase $(X)$ are situated at the same 0 and the total diffracted intensity $I_{X}(\varphi, 0)$ is given by the summation over all these layers. Each contribution is then calculated by (8).

Let us treat the case of a more usual multilayer, formed by the stacking of $A$ and $B$ phases. The sample is composed of $J$ units $A B$. B is the first deposited layer and $A$ the last one. In such a configuration, for nonoverlapped $A$ or $B$ peaks and with constant $e_{A}$ and $e_{B}$ one can have, by simple summation,

$$
\begin{aligned}
I_{A}(\varphi, \theta)= & K_{A}(\varphi, \theta)\left\{1+\sum_{i=1}^{J-1} \exp \left[-2 i\left(\mu_{A} e_{A}+\mu_{B} e_{B}\right)\right.\right. \\
& \left.\left.\times\left(\sin \theta_{A} \cos \varphi\right)^{-1}\right]\right\}
\end{aligned}
$$

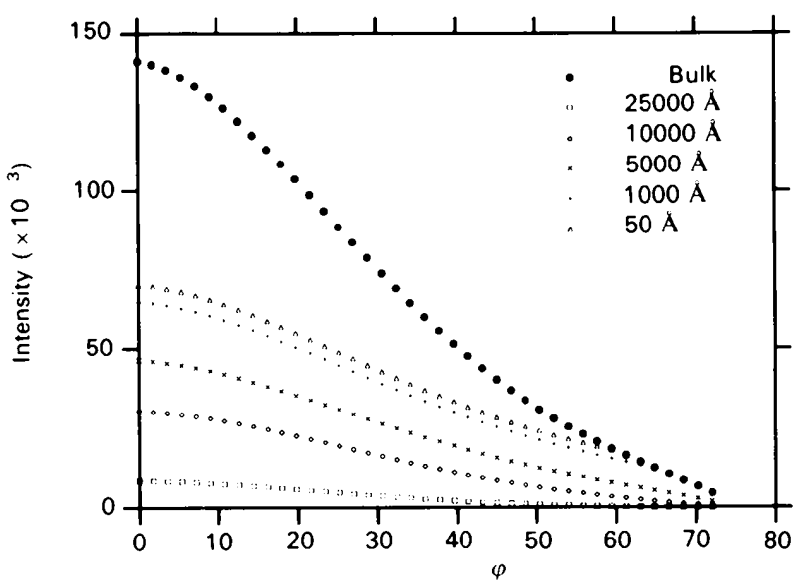

(a)

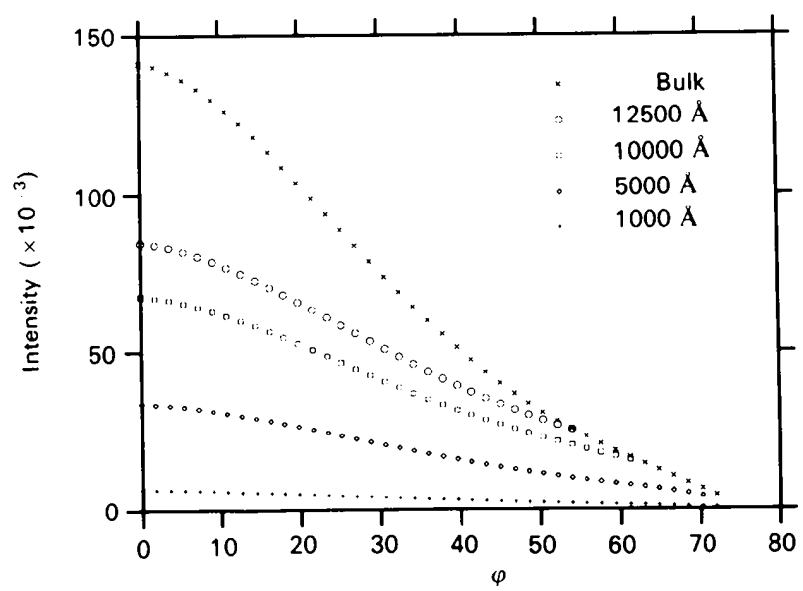

(b)

Fig. 5. Experimental bulk defocusing curve of $111 \mathrm{YSZ}$ reflection and calculated ones at $\theta=15.02 \%(a)$ for a $10000 \AA$-thick buffer layer of $\mathrm{YSZ}$ covered by $\mathrm{YBa}_{2} \mathrm{Cu}_{3} \mathrm{O}_{7}$ films of various thick nesses and $(b)$ for different thicknesses of the YSZ buffer layer covered by a $500 \AA$-thick $\mathrm{YBa}_{2} \mathrm{Cu}_{3} \mathrm{O}_{7}$ film. and

$$
\begin{aligned}
I_{\mathrm{B}}(\varphi, \theta)= & K_{B}(\varphi, \theta) \sum_{i=1}^{J} \exp \left\{-2\left[i \mu_{A} e_{A}+(i-1) \mu_{B} e_{B}\right]\right. \\
& \left.\times\left(\sin \theta_{B} \cos \varphi\right)^{-1}\right\}
\end{aligned}
$$

with

$$
K_{X}(\varphi, \theta)=e_{X} I_{X}^{\mathrm{bb}}\left(\varphi, \theta_{X}\right) / \xi_{m_{X}} \sin \theta_{X} \cos \varphi,
$$

where the subscript $X$ is $A$ or $B$, representing the two phases $A$ and $B$, respectively, and $\zeta_{m_{\mathrm{v}}}=1 / 2 \mu_{x}$

When more than two phases enter the stack, the same development is suitable for each $X$ phase. For this reason, we index the phases by the integer $k$, from 1 for the top phase $A$ to $x$ for phase $X$, to $K$ for the bottom phase of a stacked unit. It is now possible to

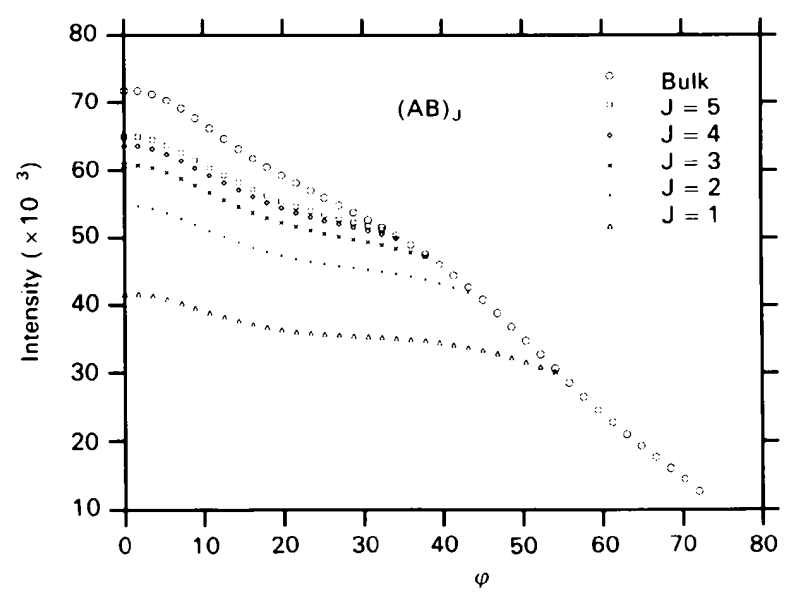

(a)

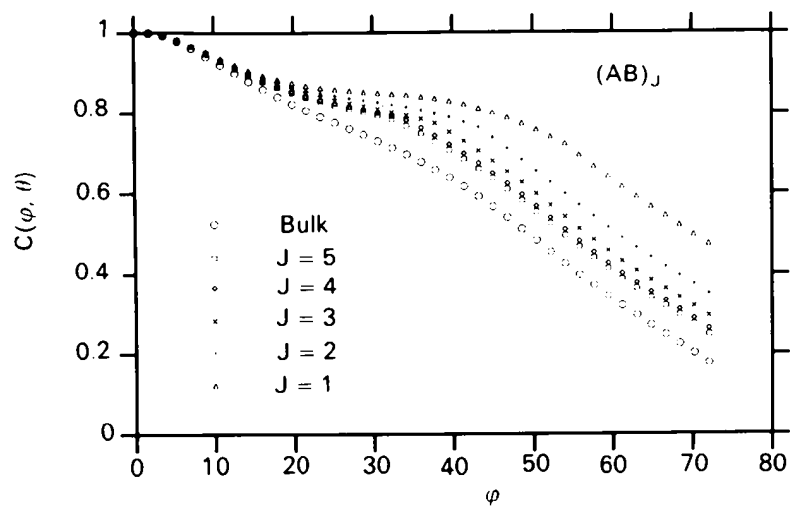

(b)

Fig. 6. Example of $(a)$ defocusing and $(b)$ correction curves for a $\left(\mathrm{YBa}_{2} \mathrm{Cu}_{3} \mathrm{O}_{7} / B\right)_{J}$ multilayer for the 103 reflection of $\mathrm{YBa}_{2} \mathrm{Cu}_{3} \mathrm{O}_{7}$ at $\theta=16.44$, from $J=0$ to 5 . 
derive the correction formula, for constant $e_{x}$ :

$$
\begin{aligned}
I_{X}(\varphi, \theta)= & K_{X}(\varphi, \theta) \\
& \times\left(\delta_{X}+\sum_{i=1}^{J-\delta_{x}} \exp \left\{\left(-2 / \sin \theta_{X} \cos \varphi\right)\right.\right. \\
& \left.\left.\times\left[i \sum_{k=1}^{x-1} \mu_{k} e_{k}+\left(i-1+\delta_{X}\right) \sum_{k=x}^{K} \mu_{k} e_{k}\right]\right\}\right)
\end{aligned}
$$

with $\delta_{X}=1$ if $k=1$ and $\delta_{X}=0$ for $k>1$.

The same remark as for buffer-layer corrections can be made about the domain where $I_{X}(\varphi, \theta)<$ $I_{X}^{\mathrm{bb}}\left(\varphi, \theta_{X}\right)$. This expression is more general than (9) and (10) since it allows the correction for both the first and last layers and for an unlimited number of different phases of the stacked unit.

Fig. 6(a) shows the $103 \mathrm{YBa}_{2} \mathrm{Cu}_{3} \mathrm{O}_{7}$ defocusing curve for a multilayer sample consisting of $J$ deposited $A B$ units. Here, $A$ is $7500 \AA$ thick $\mathrm{YBa}_{2} \mathrm{Cu}_{3} \mathrm{O}_{7}$ and $B$ is a hypothetical $0.5 \mu \mathrm{m}$-thick phase with $\mu_{B}=0.0667 \mu \mathrm{m}^{-1} . J$ varies from 1 to 5 , after which no significant differences arise between the $J$ and $(J-1)$ curves. The diffracted intensity increases with $J$, resulting in a lower $\varphi_{l}$ and a higher defocusing. On the other hand, $k$ is higher and the defocusing is lower for a specific diffraction peak, since the beam path becomes more important between two $X$ phases and $k$ increases.

For each type of sample, these curves have to be calculated including correction curves, which are drawn on Fig. $6(b)$. In this figure, points $\varphi<\varphi_{l}$ were calculated with (12) and $C(\varphi, \theta)$ differs a lot from the bulk. The points after $\varphi_{l}$ were translated from the bulk curve, accounting for the difference in the irradiated material volume. From these curves, accurate correction of pole figures is possible.

\section{Concluding remarks}

We have derived the principal limits in thin sample texture analysis and drawn attention to their practical consequences for the defocusing correction of pole figures. The effects on the correction curves have been reviewed using some precise examples. The correction of covered substrates has also been described.

The Alcatel-Alsthom Research Group (Laboratoires de Marcoussis, France) is gratefully acknowledged for its partial support of this work.

\section{References}

Chateigner, D., Germi, P. \& Pernet, M. (1992). J. Appl. Cryst. 25, 766-769.

Coutfrne, J. C. \& Cizeron, G. (1971). J. Appl. Cryst. 4, 461-472.

Gale, B. \& Griffiths, D. (1960). Br. J. Appl. Phys. 11, 96-102.

Reller, A., Bednorz, J. \& Muller, K. (1989). Powder Diffraction File No. 40-159, International Centre for Diffraction Data, Swarthmore, PA, USA.

Schulz, L. G. (1949). J. Appl. Phys. 20, 1030-1037.

Tenckhoff, E. (1970). J. Appl. Phys. 41, 3944-3948.

Wenk, H. R., Sintubin, M., Huang, J., Johnson, G. C. \& Howe, R. T. (1990). J. Appl. Phys. 67, 572-574. 